\title{
Research Article \\ Complex Dynamic Behaviors in Cellular Automata Rule 14
}

\section{Qi Han, Xiaofeng Liao, and Chuandong Li}

State Key Laboratory of Power Transmission Equipment and System Security, College of Computer Science, Chongqing University, Chongqing 400030, China

Correspondence should be addressed to Qi Han, yiding1981@yahoo.com.cn

Received 2 November 2011; Revised 1 February 2012; Accepted 1 February 2012

Academic Editor: Vimal Singh

Copyright (C) 2012 Qi Han et al. This is an open access article distributed under the Creative Commons Attribution License, which permits unrestricted use, distribution, and reproduction in any medium, provided the original work is properly cited.

Wolfram divided the 256 elementary cellular automata rules informally into four classes using dynamical concepts like periodicity, stability, and chaos. Rule 14 , which is Bernoulli $\sigma_{\tau}$-shift rule and is a member of Wolfram's class II, is said to be simple as periodic before. Therefore, it is worthwhile studying dynamical behaviors of rule 14, whether it possesses chaotic attractors or not. In this paper, the complex dynamical behaviors of rule 14 of one-dimensional cellular automata are investigated from the viewpoint of symbolic dynamics. We find that rule 14 is chaotic in the sense of both Li-Yorke and Devaney on its attractor. Then, we prove that there exist fixed points in rule 14. Finally, we use diagrams to explain the attractor of rule 14, where characteristic function is used to describe that all points fall into Bernoulli-shift map after two iterations under rule 14.

\section{Introduction}

Cellular automata (CA) was first introduced by von Neumann in 1951 [1]. CA is a mathematical model consisting of large numbers of simple identical components with local interactions [2]. The simple components act together to produce complex global behavior. CA performs complex computation with high degree of efficiency and robustness. Therefore, many applications of CA have been reported, especially in cryptography $[3,4]$ and image processing $[5,6]$.

Here, we will only consider Boolean automata for which the cellular state $x \in\{0,1\}$. A cellular automata consists of a number of cells which evolve by a simple local rule (identical rule). The value of each cell in the next stage is determined by the values of the cell and its neighbor cells in the current stage under the local rule [4]. The identical rule contained in each cell is essentially a finite-state machine, usually specified in the form of a rule table, with an entry for every possible neighborhood of a cell consists of itself and the adjacent cells [7]. The cellular array is $d$-dimensional, where $d=1,2,3$ is used in practice. In this paper, 
Table 1: The truth table of Boolean function of rule 14.

\begin{tabular}{lccc}
\hline$x_{i-1} x_{i} x_{i+1}$ & {$\left[f_{14}(x)\right]_{i}$} & $x_{i-1} x_{i} x_{i+1}$ & {$\left[f_{14}(x)\right]_{i}$} \\
\hline 000 & 0 & 100 & 0 \\
001 & 1 & 101 & 0 \\
010 & 1 & 110 & 0 \\
011 & 1 & 111 & 0 \\
\hline
\end{tabular}

we will concentrate on $d=1$. For a one-dimensional CA, a cell is connected to $r$ local neighbors (cells) on either side where $r$ is referred to as the radius. A one-dimensional CA has $n$ cells linked in a line or in a circle. Denote the value in the $i$ th cell at the $t$ th stage by $x_{i}[t]$. For 2-state 3-neighborhood CA $(r=1)$, the evolution of $i$ th cell can be represented as a function of the present states of $(i-1)$ th, $(i)$ th, and $(i+1)$ th. The local function $f_{i}$ is a deterministic function to determine the next-stage value of the $i$ th cell, $x_{i}[t+1]=f_{i}\left(x_{i-1}[t], x_{i}[t], x_{i+1}[t]\right)$. For example, the rule 14 is a one-dimensional CA, and its rule table is shown in Table 1 . Thus, we have $f(000)=0, f(001)=1, f(010)=1, f(011)=1, f(100)=0, f(101)=0, f(110)=0$, and $f(111)=0$.

In 1980s, Wolfram proposed CA as models for physical systems which exhibit complex or even chaotic behaviors based on empirical observations, and he divided the 256 ECA (binary one-dimensional CA with radius 1) rules informally into four classes using dynamical concepts like periodicity, stability, and chaos [8-10]. However, some authors [11-14] found that some rules of Bernoulli $\sigma_{\tau}$-shift rules are chaotic in the sense of both Li-York and Devaney, where these rules were said to be simple as periodic by Wolfram. Rule 24 is belonging to Bernoulli $\sigma_{\tau}$-shift rules. Therefore, we need to research the rule 24 and to find its some new dynamical properties. The rest of the paper is organized as follows. In Section 2, the Boolean function of rule 14 is also presented, and the expressions of its attractors are given. In Section 3, the dynamical behaviors of rule 14 are studied. In Section 4, characteristic function is used to describe that all points fall into Bernoulli-shift map after several iterations under rule 14, and Lameray diagram is used to show clearly the iterative process of an attractor. Section 5 presents some conclusions.

\section{Preliminaries}

For simplicity, some notations about symbolic dynamics can refer to $[11,14]$.

In this paper, we will use some notations about CA as follows.

Chua et al. [15] mentioned that each rule has three globally equivalent local rules determined by three corresponding global transformations, namely, left-right transformation $T^{\dagger}$, global complementation $\bar{T}$, and left-right complementation $T^{*}$. Each equivalence class is identified by $\varepsilon_{m}^{\kappa}$, where $\kappa$ is complexity index, and $m$ is index of $\kappa$ th class. In [16], the authors presented that 112 rules of 256 local rules were Bernoulli $\sigma_{\tau}$-shift rules. Each of the 112 Bernoulli $\sigma_{\tau}$-shift rules has an ID code $B_{N}[\alpha, \beta, \tau]$, where $\alpha$ denotes the number of attractors of rule $N, \beta$ denotes the slope of the Bernoulli $\sigma_{\tau}$-shift map, and $\tau$ denotes the relevant forward time- $\tau$. Hence, the space-time evolution of any one of the 112 rules on their attractors can be uniquely predicted by two parameters: $\beta= \pm 2^{\sigma}$ and $\tau$. For example, the attractors of rule 14 are $(\beta=-1 / 2, \sigma=-1, \tau=1)$ and $(\beta=2, \sigma=1, \tau=1)$.

It follows from [17] that the Boolean function of rule 14 is

$$
\left[f_{14}(x)\right]_{i}=\bar{x}_{i-1} \cdot x_{i} \oplus \bar{x}_{i-1} \cdot \bar{x}_{i} \cdot x_{i+1}
$$


for $\forall x \in S^{Z}, i \in Z$, where ".", "-", and " $\oplus$ " stand for "AND", "NOT", and "XOR" logical operation, respectively. Sometimes, "." is omitted for simplicity. The truth table of Boolean function of rule 14 is shown in Table 1.

The subsets denoted by $\Lambda_{1}^{14}, \Lambda_{2}^{14}$ are derived from the parameters of rule $14: \beta=-1 / 2$, $\sigma=-1$, and $\tau=1$ and $\beta=2, \sigma=1$, and $\tau=1$, respectively, that is,

$$
\begin{aligned}
& \Lambda_{1}^{14}=\left\{x \in S^{Z} \mid\left[f_{14}(x)\right]_{i}=\bar{x}_{i-1}, \forall i \in Z\right\}, \\
& \Lambda_{2}^{14}=\left\{x \in S^{Z} \mid\left[f_{14}(x)\right]_{i}=x_{i+1}, \forall i \in Z\right\} .
\end{aligned}
$$

\section{Dynamical Behaviors of $f_{14}$ on Two Subsystems}

In this section, we investigate the complexity and chaotic dynamic behaviors of $f_{14}$. In order to give our results, some definitions need be introduced.

Definition 3.1 (see [18]). A square $\{0,1\}$ matrix $A$ is irreducible if for every pair of indices $i$ and $j$, there is an $n$ such that $A_{i j}^{n}>0$.

Definition 3.2 (see [18]). A square $\{0,1\}$ matrix $A$ is aperiodic if there exists $N$, such that $A_{i j}^{n}>0, n>N, \forall i, j$.

Definition 3.3 (see [18]). Suppose that $g: X \rightarrow Y$ is a continuous mapping, where $X$ is a compact topological space. $g$ is said to be topologically mixing if, for any two open sets $U$, $V \subset X, \exists N>0$, such that $g^{n}(U) \cap V \neq \emptyset, \forall n \geq N$.

Definition 3.4 (see [13]). Let $(X, f)$ and $(Y, g)$ be compact spaces, we say $f$ and $g$ are topologically conjugate if there is a homeomorphism $h: X \rightarrow Y$, such that $h \circ f=g \circ h$.

Based on the above definitions, we investigate the complexity behaviors of $f_{14}$ on two subsystems as follows.

Proposition 3.5. For rule 14, there exists a subset $\Lambda_{1}^{14} \subset S^{Z}$ which satisfies $\left.f_{14}\right|_{\Lambda_{1}^{14}}=S_{1}^{14} \circ$ $\left.\bar{T}\right|_{\Lambda_{1}^{14}}=\left.\Gamma_{14}\right|_{\Lambda_{1}^{14}}$ if and only if $\forall x=\left(\ldots, x_{-1}, x_{0}, x_{1}, \ldots\right) \in \Lambda_{1}^{14}$, and $x_{i-1}, x_{i}$, and $x_{i+1}$ cannot be 0 simultaneously, $\forall i \in Z$.

Proof. (Necessity) Suppose that there exists a subset $\Lambda_{1}^{14} \in S^{Z}$ such that $\left.f_{14}\right|_{\Lambda_{1}^{14}}=\left.\Gamma_{14}\right|_{\Lambda_{1}^{14}}$, then $\forall x=\left(\ldots, x_{-1}, x_{0}, x_{1}, \ldots\right) \in \Lambda_{1}^{14}$, and we have $\left[f_{14}(x)\right]_{i}=\bar{x}_{i-1}, \forall x \in Z$.

If $x_{i}=1$, then $\bar{x}_{i-1} x_{i} \oplus \bar{x}_{i-1} \bar{x}_{i} x_{i+1}=1 \oplus x_{i-1} \oplus 0=\bar{x}_{i-1}$, so we get $x_{i-1}=0, x_{i+1}=0 ; x_{i-1}=0$, $x_{i+1}=1 ; x_{i-1}=1, x_{i+1}=0 ; x_{i-1}=1, x_{i+1}=1$; if $x_{i}=0$, then $\bar{x}_{i-1} x_{i} \oplus \bar{x}_{i-1} \bar{x}_{i} x_{i+1}=0 \oplus \bar{x}_{i-1} x_{i+1}$, so we get $x_{i+1}=1, x_{i-2}=1$; if $x_{i-1}=1$, then $x_{i+1}=1$ or $x_{i+1}=0$.

(Sufficiency) Suppose that there exists a subset $\Lambda_{1}^{14} \subset S^{Z}, \forall x \in \Lambda_{1}^{14}, x_{i-1}, x_{i}$, and $x_{i+1}$ cannot be 0 simultaneously, $\forall i \in Z$. This yields that $x_{i-1}+x_{i}+x_{i+1}=1$. Thus, $\left(x_{i-1}+x_{i}+x_{i+1}\right) \oplus$ $x_{i-1}=\bar{x}_{i-1}$, namely, $\bar{x}_{i-1} x_{i} \oplus \bar{x}_{i-1} \bar{x}_{i} x_{i+1}=\bar{x}_{i-1}$. Therefore, we have proven that $f_{14}(x)=\Gamma_{14}(x)$, $\forall x \in \Lambda_{1}^{14}$.

Remark 3.6. A condition of bit strings according to Bernoulli $\sigma_{\tau}$-shift evolution under $f_{14}$ is obtained in Proposition 3.5. From Proposition 3.5, we can know that a bit string belonging 
to $\Lambda_{1}^{14}$ is evolved on the basis of shifting the bit string to the right by 1 bit and then complementing (changing 0 to 1 and changing 1 to 0 ) to obtain the next bit string after one iteration under $f_{14}$.

Remark 3.7. From the definition of subsystem, we know that $\left(\Lambda_{1}^{14}, f_{14}\right)$ are subsystems of $\left(S^{Z}, f_{14}\right)$.

Proposition 3.8. $\Gamma_{14}=\varsigma_{14} \circ \bar{T}=\bar{T} \circ \varsigma_{14}$.

Proposition 3.9. $\varsigma_{14}: S^{Z} \rightarrow S^{Z}$ and $\Gamma_{14}: S^{Z} \rightarrow S^{Z}$ are not topologically conjugate.

$\left(\Lambda_{1}^{14}, \varsigma_{14}\right)$ is topologically mixing if and only if $\left(\Lambda_{1}^{14}, \Gamma_{14}\right)$ is topologically mixing.

The topological entropy ent $\left(\left.\&_{14}\right|_{\Lambda_{1}^{14}}\right)=\operatorname{ent}\left(\left.\Gamma_{14}\right|_{\Lambda_{1}^{14}}\right)$.

Proof. (a) Suppose that $\varsigma_{14}$ and $\Gamma_{14}$ are topologically conjugate, we give a proof by contradiction. Since $\varsigma_{14}$ and $\Gamma_{14}$ are topologically conjugate, then there exists a homeomorphism $h$ such that $h \circ \varsigma_{14}=\Gamma_{14} \circ h$, so we have $h \circ \varsigma_{14}=\bar{T} \circ \varsigma_{14} \circ h$. Because of $h \circ \varsigma_{14}=\varsigma_{14} \circ h$, then we obtain that $\bar{T}$ is identical map, which leads to a contradiction.

(b) Let any nonempty open sets $U, V \subset \Lambda_{1}^{14}$.

(Sufficiency) Assume that $\left.\Gamma_{14}\right|_{\Lambda_{1}^{14}}$ is topologically mixing, then there exists $N_{1}>0$, such that $\left(\left.\Gamma_{14}\right|_{\Lambda_{1}^{14}}\right)^{n}(U) \cap V \neq \emptyset, \forall n \geq N_{1}$. Since $\bar{T}$ is a homeomorphism, $\bar{T}(V)$ is also a nonempty open set in $\Lambda_{1}^{14}$, hence there exists $N_{2}>0$, such that $\left(\left.\Gamma_{14}\right|_{\Lambda_{1}^{14}}\right)^{n}(U) \cap \bar{T}(V) \neq \emptyset, \forall n \geq N_{2}$. Therefore, $\bar{T}^{-1}\left(\left(\left.\Gamma_{14}\right|_{\Lambda_{1}^{14}}\right)^{n}(U) \cap \bar{T}(V)\right) \neq \emptyset, \forall n \geq N_{2}$. Then, we show that there exists a $N>0$ such that $\left(\left.\varsigma_{14}\right|_{\Lambda_{1}^{14}}\right)^{n}(U) \cap V \neq \emptyset, \forall n \geq N$. Let $N=\max \left(N_{1}, N_{2}\right)$.

(i) If $n=2 k, k \in Z^{+}$, then

$$
\begin{aligned}
& \left(\left.s_{14}\right|_{\Lambda_{1}^{14}}\right)^{n}(U) \cap V=\bar{T}^{n} \circ\left(\left.s_{14}\right|_{\Lambda_{1}^{14}}\right)^{n}(U) \cap V
\end{aligned}
$$

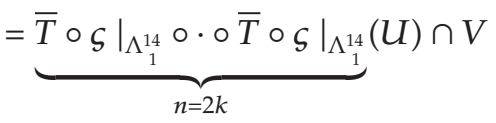

$$
\begin{aligned}
& =\left(\left.\Gamma_{14}\right|_{\Lambda_{1}^{14}}\right)^{n}(U) \cap V \neq \emptyset \text {. }
\end{aligned}
$$

(ii) If $n=2 k+1, k \in Z^{+}$, then

$$
\begin{aligned}
\left(\left.s_{14}\right|_{\Lambda_{1}^{14}}\right)^{n}(U) \cap V & =\overline{\mathrm{T}}^{-1} \circ \bar{T}\left(\left(\left.s_{14}\right|_{\Lambda_{1}^{14}}\right)^{n}(U) \cap V\right) \\
& =\overline{\mathrm{T}}^{-1} \circ\left(\bar{T} \circ\left(\left.s_{14}\right|_{\Lambda_{1}^{14}}\right)^{n}(U) \cap \bar{T}(V)\right) \\
& =\overline{\mathrm{T}}^{-1} \circ\left(\left(\left.\Gamma_{14}\right|_{\Lambda_{1}^{14}}\right)^{n}(U) \cap \bar{T}(V)\right) \neq \emptyset .
\end{aligned}
$$

(Necessity) Suppose that $\left.\varsigma_{14}\right|_{\Lambda_{1}^{14}}$ is topologically mixing, then there exists an $N_{1}>0$, such that $\left(\left.\varsigma_{14}\right|_{\Lambda_{1}^{14}}\right)^{n}(U) \cap V \neq \emptyset, \forall n \geq N_{1}$. Since $\bar{T}$ is a homeomorphism, $\bar{T}(U)$ is also a nonempty open set in $\Lambda_{1}^{14}$, hence for open sets $\bar{T}(U)$ and $V$, there exists an $N_{2}>0$, such that $\left(\left.\varsigma_{14}\right|_{\Lambda_{1}^{14}}\right)^{n} \circ \bar{T}(U) \cap V \neq \emptyset$. Let $N=\max \left(N_{1}, N_{2}\right)$. 
(i) If $n=2 k, k \in Z^{+}$, then

$$
\begin{aligned}
\left(\left.\Gamma_{14}\right|_{\Lambda_{1}^{14}}\right)^{n}(U) \cap V & =\bar{T}^{n} \circ\left(\left.s_{14}\right|_{\Lambda_{1}^{14}}\right)^{n}(U) \cap V \\
& =\left(\left.s_{14}\right|_{\Lambda_{1}^{14}}\right)^{n}(U) \cap V \neq \emptyset .
\end{aligned}
$$

(ii) If $n=2 k+1, k \in Z^{+}$, then

$$
\begin{aligned}
\left(\left.\Gamma_{14}\right|_{\Lambda_{1}^{14}}\right)^{n}(U) \cap V & =\bar{T} \circ\left(\left.s_{14}\right|_{\Lambda_{1}^{11}}\right)^{n}(U) \cap V \\
& =\left(\left.s_{14}\right|_{\Lambda_{1}^{14}}\right)^{n} \circ \overline{\mathrm{T}}(U) \cap V \neq \emptyset .
\end{aligned}
$$

(c) $\left(\left.\Gamma_{14}\right|_{\Lambda_{1}^{14}}\right)^{2}=\left(\left.\bar{T} \circ \varsigma_{14}\right|_{\Lambda_{1}^{14}}\right)^{2}=(\bar{T})^{2} \circ\left(\left.\varsigma_{14}\right|_{\Lambda_{1}^{14}}\right)^{2}=\left(\left.\varsigma_{14}\right|_{\Lambda_{1}^{14}}\right)^{2}$.

Hence, $\operatorname{ent}\left(\left.\varsigma_{14}\right|_{\Lambda_{1}^{14}}\right)=\operatorname{ent}\left(\left.\Gamma_{14}\right|_{\Lambda_{1}^{14}}\right)$. The proof is complete.

Because of $\left.f_{14}\right|_{\Lambda_{1}^{14}}=\left.\Gamma_{14}\right|_{\Lambda_{1}^{14}}$, the following corollaries are immediate.

Corollary 3.10. $\left.\varsigma_{14}\right|_{\Lambda_{1}^{14}}$ and $\left.f_{14}\right|_{\Lambda_{1}^{14}}$ are not topologically conjugate.

Corollary 3.11. $\left(\Lambda_{1}^{14}, \varsigma_{14}\right)$ is topologically mixing if and only if $\left(\Lambda_{1}^{14}, f_{14}\right)$ is topologically mixing.

Corollary 3.12. The topological entropy $\operatorname{ent}\left(\left.\&_{14}\right|_{\Lambda_{1}^{14}}\right)=\operatorname{ent}\left(\left.f_{14}\right|_{\Lambda_{1}^{14}}\right)$.

Remark 3.13. By Proposition 3.5, we know that the determinative block system of $\Lambda_{1}^{14}$ is $P_{1}=$ $\left\{r_{0}, r_{1}, r_{2}, r_{3}, r_{4}, r_{5}, r_{6}\right\}$, where $r_{0}=(001), r_{1}=(010), r_{2}=(011), r_{3}=(100), r_{4}=(101), r_{5}=(110)$, and $r_{6}=(111)$. We have $\Lambda_{1}^{14}=\Lambda_{P_{1}}$.

Let $\varpi_{1}=\left\{\left(r r^{\prime}\right) \mid r=\left(b_{0} b_{1} b_{2}\right), r^{\prime}=\left(b_{0}^{\prime} b_{1}^{\prime} b_{2}^{\prime}\right) \in P_{1}, \exists 1 \leq j \leq 2\right.$ such that $\left.b_{j} \neq b_{j-1}^{\prime}\right\} . \varpi_{1}$ is considered as the excluded block system [19] $\Lambda_{\varpi_{1}}$, where $\Lambda_{\varpi_{1}}=\left\{r=\left(\ldots r_{-1}, r_{0}, r_{1} \ldots\right) \in\right.$ $\left.P_{1} \mid r_{i} \in P_{1}, r_{i} r_{i+1} \nless \varpi_{1}, \forall i \in Z\right\}$. Obviously, $\varsigma_{14}: \Lambda_{\varpi_{1}} \rightarrow \Lambda_{\varpi_{1}}, \varsigma_{14}\left(\ldots, r_{-1} \widehat{r}_{0} r_{1}, \ldots\right) \rightarrow$ $\left(\ldots, \widehat{r}_{-1} r_{0} r_{1}, \ldots\right)$. Therefore, it is easy to calculate the transition matrix $B^{1}$ of the $\left.\varsigma\right|_{\Lambda_{\sigma_{1}}}$ as

$$
B^{1}=\left[\begin{array}{lllllll}
0 & 1 & 1 & 0 & 0 & 0 & 0 \\
0 & 0 & 0 & 1 & 1 & 0 & 0 \\
0 & 0 & 0 & 0 & 0 & 1 & 1 \\
1 & 0 & 0 & 0 & 0 & 0 & 0 \\
0 & 1 & 1 & 0 & 0 & 0 & 0 \\
0 & 0 & 0 & 1 & 1 & 0 & 0 \\
0 & 0 & 0 & 0 & 0 & 1 & 1
\end{array}\right],
$$




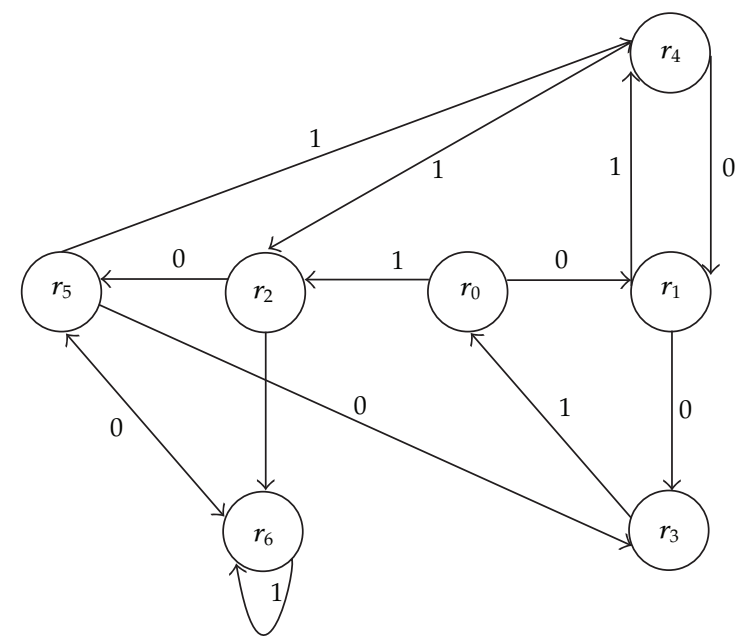

Figure 1: The corresponding graph $G_{1}^{14}$ of the matrix $B^{1}$.

where $B^{1}=\left(B_{i j}^{1}\right)_{\left|P_{1}\right| \times\left|P_{1}\right|}$

$$
B_{i j}^{1}= \begin{cases}1, & r_{i} r_{j} \prec \varpi_{1} \\ 0, & \text { else. }\end{cases}
$$

Obviously, $B^{1}$ is a square $\{0,1\}$ matrix. A square $\{0,1\}$ matrix corresponds to a directed graph. The vertices of the graph are the indices for the rows and columns of $B^{1}$. There is an edge from vertex $i$ to vertex $j$ if $B_{i j}^{1}=1$. A square $\{0,1\}$ matrix $A$ is irreducible if and only if the corresponding graph is strongly connected. If $\Lambda_{A}$ is a two-order subshift of finite type, then it is topologically mixing if and only if $A$ is irreducible and aperiodic [18].

We give the corresponding graph $G_{1}^{14}$ of matrix $B^{1}$ in Figure 1, where vertices are the elements of set $P_{1}$. It is obvious that $G_{1}^{14}$ is a strongly connected graph.

Carefully observing Figure 1 , we find that there are several strongly connected subgraphs: $r_{0} \rightarrow r_{1} \rightarrow r_{3} \rightarrow r_{0}, r_{0} \rightarrow r_{1} \rightarrow r_{4} \rightarrow r_{1} \rightarrow r_{3} \rightarrow r_{0}, r_{0} \rightarrow r_{2} \rightarrow r_{5} \rightarrow r_{3} \rightarrow r_{0}$, $r_{0} \rightarrow r_{2} \rightarrow r_{6} \rightarrow r_{5} \rightarrow r_{4} \rightarrow r_{1} \rightarrow r_{3} \rightarrow r_{0}, r_{1} \rightarrow r_{4} \rightarrow r_{1}, r_{2} \rightarrow r_{5} \rightarrow r_{4} \rightarrow r_{2}$, $r_{2} \rightarrow r_{6} \rightarrow r_{5} \rightarrow r_{4} \rightarrow r_{2}, r_{6} \rightarrow r_{6}$, and so forth. The elements of $\Lambda_{P_{1}}^{14}$ will be composed by all vertices of those strongly connected subgraphs, respectively. For example, if $x_{1} \in \Lambda_{P_{1}}^{14}$, $x_{1}$ is composed by vertices of subgraph $r_{1} \rightarrow r_{4} \rightarrow r_{1}$, then we have $r_{0}, r_{2}, r_{3}, r_{5}$, and $r_{6} \nless x_{1}$, and all vertices of the subgraph will occur in $x_{1}$ if $\left|x_{1}\right|=2 k, k=1,2, \ldots$ If $x_{2} \in \Lambda_{P_{1}}^{142}$ and $x_{2}$ is composed by vertices of subgraph $r_{2} \rightarrow r_{5} \rightarrow r_{4} \rightarrow r_{2}$, then we have $r_{0}, r_{1}, r_{3}$, and $r_{6} \nless x_{2}$, and all vertices of the subgraph will occur in $x_{2}$ if $\left|x_{2}\right|=3 k$. Therefore, we can deduce the elements of set $\Lambda_{1}^{14}$ via the corresponding strongly connected graph of matrix $B^{1}$.

Based on the above analysis, we have the following proposition.

Proposition 3.14. (a) $\varsigma_{14}: \Lambda_{1}^{14} \rightarrow \Lambda_{1}^{14}$ and $\varsigma_{14}: \Lambda_{\varpi_{1}} \rightarrow \Lambda_{\varpi_{1}}$ are topologically conjugate.

(b) $\left.\varsigma_{14}\right|_{\Lambda_{1}^{14}}$ is topologically mixing.

(c) The topological entropy ent $\left(\left.\&_{14}\right|_{\Lambda_{1}^{14}}\right)=0.6094$. 
Proof. (a) Define

$$
\begin{gathered}
h: \Lambda_{1}^{14} \longrightarrow \Lambda_{\varpi_{1}}, \\
x=\left(\ldots, x_{-1}, \stackrel{*}{x}_{0}, x_{1}, \ldots\right) \longmapsto\left(\ldots, r_{-1}, \stackrel{*}{r}_{0}, r_{1}, \ldots\right),
\end{gathered}
$$

where $r_{i}=\left(x_{i} x_{i+1} x_{i+2}\right), \forall i \in Z$. In fact, by the definition of $\Lambda_{\varpi_{1}}$, we have $h(x) \in \Lambda_{\varpi_{1}}, \forall x \in \Lambda_{1}^{14}$; thus, $h\left(\Lambda_{1}^{14}\right) \subseteq h\left(\Lambda_{\varpi_{1}}\right)$. Then, it is easy to check that $h$ is homeomorphism and $\left.h \circ \varsigma_{14}\right|_{\Lambda_{1}^{14}} \rightarrow$ $\left.\varsigma_{14}\right|_{\Lambda_{\pi_{1}}} \circ h$. Therefore, $\left.\varsigma_{14}\right|_{\Lambda_{1}^{14}}$ and $\left.\varsigma_{14}\right|_{\Lambda_{\omega_{1}}}$ are topologically conjugate.

(b) Because $\left(B^{1}\right)^{n}>0, \forall n \geq 4$, the transition matrix $B^{1}$ of subshift of finite type $\varsigma$ is irreducible and aperiodic. By $[18,19],\left.\varsigma_{14}\right|_{\Lambda_{1}^{14}}$ is topologically mixing.

(c) Because two topological conjugate systems have the same topological entropy, and the topological entropy $\varsigma_{14}$ on $\Lambda_{\varpi_{1}}$ equals $\log \rho\left(B^{1}\right)$, where $\rho\left(B^{1}\right)$ is the spectral radius of the transition matrix $B_{1}^{14}$ of the subshift $\Lambda_{\varpi_{1}}$. So $\operatorname{ent}\left(\left.\varsigma_{14}\right|_{\Lambda_{1}^{14}}\right)=\log \rho\left(B^{1}\right)=0.6094$.

Remark 3.15. By Corollaries 3.11 and 3.12, we know that $\left(\Lambda_{1}^{14}, f_{14}\right)$ is topologically mixing and $\operatorname{ent}\left(\left.f_{14}\right|_{\Lambda_{1}^{14}}\right)=0.6094$

Theorem 3.16. $f_{14}$ is chaotic in the sense of both Li-Yorke and Devaney on $\Lambda_{1}^{14}$.

Proof. It follows from [19] that the positive topological entropy implies chaos in the sense of Li-Yorke, and a system with topologically mixing property has chaotic properties in different senses such as Devaney. Therefore, rule $N=14$ possesses very rich and complicated dynamical properties on $\Lambda_{1}^{14}$.

Proposition 3.17. For rule 14, there exists a subset $\Lambda_{2}^{14} \subset S^{Z}$ which satisfies $\left.f_{14}\right|_{\Lambda_{2}^{14}}=\left.v_{14}\right|_{\Lambda_{2}^{14}}$ if and only if $\forall x=\left(\ldots, x_{-1}, x_{0}, x_{1}, \ldots\right) \in \Lambda_{2}^{14}$, and $x_{i-1}, x_{i}$, and $x_{i+1}$ have the following relations:

(i) if $x_{i}=1$, then $x_{i-1}=0, x_{i+1}=1$, and $x_{i+2}=0 ; x_{i-1}=1, x_{i+1}=0$, and $x_{i+2}=0$,

(ii) if $x_{i}=0$, then $x_{i-1}=0, x_{i+1}=0 ; x_{i-1}=1, x_{i+1}=0 ; x_{i-1}=0, x_{i+1}=1$, and $x_{i+2}=1$.

Let $P_{2}=\left\{r_{0}, r_{1}, r_{2}, r_{3}, r_{4}\right\}$ be a new state set, where $r_{0}=(000), r_{1}=(001), r_{2}=(011)$, $r_{3}=(100)$, and $r_{4}=(110)$.

Remark 3.18. From the definition of subsystem, we know that $\left(\Lambda_{2}^{14}, f_{14}\right)$ is subsystem of $\left(S^{Z}, f_{14}\right)$.

The transition matrix of subshift $\left(\Lambda_{2}^{142}, v_{14}\right)$ is

$$
B^{2}=\left[\begin{array}{lllll}
1 & 1 & 0 & 0 & 0 \\
0 & 0 & 1 & 0 & 0 \\
0 & 0 & 0 & 0 & 1 \\
1 & 1 & 0 & 0 & 0 \\
0 & 0 & 0 & 1 & 0
\end{array}\right] .
$$

We give the corresponding graph $G_{2}^{14}$ of matrix $B^{2}$ in Figure 2, where vertices are the elements of set $P_{2}$. 


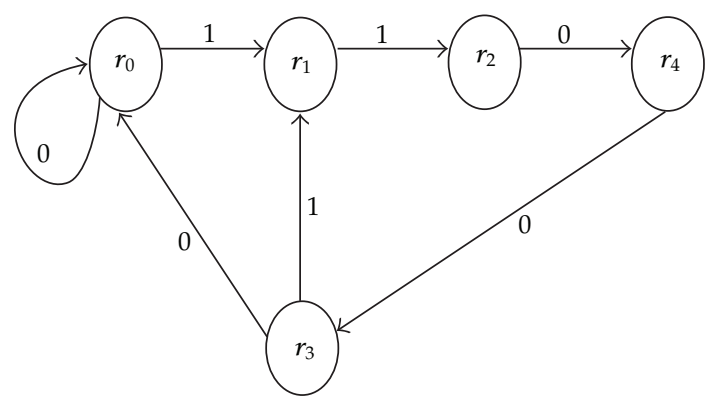

Figure 2: The corresponding graph $G_{2}^{14}$ of the matrix $B^{2}$.

Proposition 3.19. (a) $\left.f_{14}\right|_{\Lambda_{2}^{14}}$ is topologically mixing.

(b) The topological entropy $\operatorname{ent}\left(\left.f_{14}\right|_{\Lambda_{2}^{14}}\right)=0.3223$.

Theorem 3.20. $f_{14}$ is chaotic in the sense of both Li-Yorke and Devaney on $\Lambda_{2}^{14}$.

Theorem 3.21. For rule 14, there exist fixed points of $f_{14}$.

Proof. A example is given as follows. Let

$$
y=(\underbrace{\ldots 0,1,0,1,0,1}_{\text {repetition of }(0,1)}, 0,1, \stackrel{*}{0}, 1, \underbrace{0,1,0,1,0,1 \ldots}_{\text {repetition of }(0,1)}) .
$$

By Table 1, we have

$$
f_{14}(y)=(\underbrace{\ldots 0,1,0,1,0,1}_{\text {repetition of }(0,1)}, 0,1, \stackrel{*}{0}, 1, \underbrace{0,1,0,1,0,1 \ldots}_{\text {repetition of }(0,1)})=y \text {. }
$$

Therefore, there exists a set of fixed points of rule 14 .

Remark 3.22. By Theorem 3.21, we know that rule 14 has garden of Eden [20] (An bit string is said to be a garden of Eden of rule $N$ if and only if it does not have a predecessor under the local rule transformation $f_{N}$ ) in the finite case. By definition of garden of Eden, the incoming and outgoing bit strings are the same in a garden of Eden, while the basin of attraction of an attractor $\Lambda$ must contain, at least, one point not belonging to $\Lambda$. However, in this paper, we consider garden of Eden as a kind of special attractor. Then, we find that (000), (001), (010), (011), (100), (101), (110), or (111) $\prec \Lambda_{14}=\Lambda_{1}^{14} \cup \Lambda_{2}^{14}$. Furthermore, we guess that all initial bit strings after sufficient iterations will belong to $\Lambda_{14}$ under rule $f_{14}$.

\section{Characteristic Function of Rule 14}

First, we give a definition on global characteristic function [16]. Given any local rule $N$, and binary configuration $x=\left[x_{0} x_{1} \cdot x_{I-1} x_{I}\right]$ for CA, where $x_{i} \in\{0,1\}$, then we can uniquely 


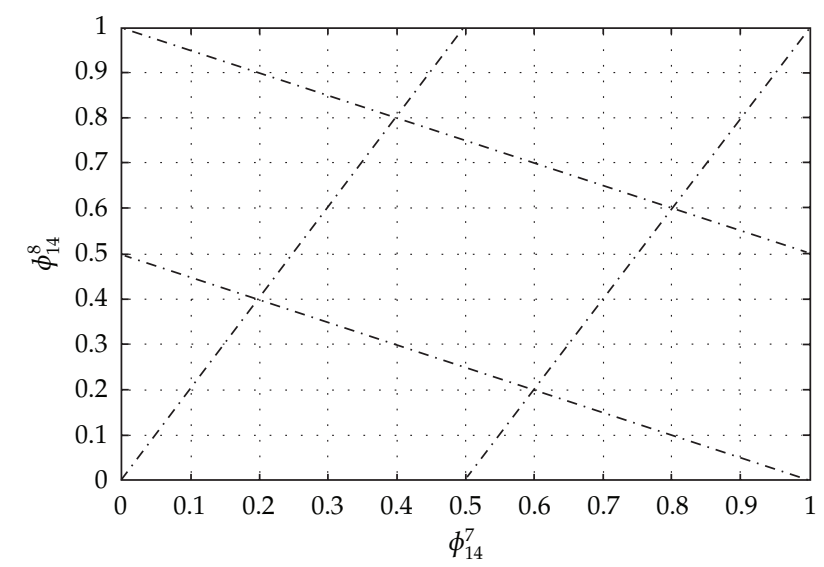

Figure 3: All points fall into Bernoulli-shift map after seven iterations under rule 14, where $I=13$.

associate the Boolean string $x$ with the binary expansion of a real number $0 . x_{0} x_{1} \cdot x_{I-1} x_{I}$ on the unit interval $[0,1]$,

$$
x=\left[x_{0} x_{1} \cdot x_{I-1} x_{I}\right] \longmapsto \phi=0 \bullet x_{0} x_{1} \cdot x_{I-1} x_{I},
$$

where $\phi=\sum_{i=0}^{I} 2^{-(i+1)} x_{i}$ is the decimal form of Boolean string $x=\left[x_{0} x_{1} \cdot x_{I-1} x_{I}\right]$. The $\mathrm{CA}^{\prime}$ characteristic function $\chi_{N}$ of rule $N$ is defined as

$$
\chi_{N}: Q[0,1] \longrightarrow Q[0,1], \text { i.e. } \phi_{n}=x_{N}^{1}\left(\phi_{n-1}\right),
$$

where $Q$ denotes rational numbers. By [19], the express of characteristic function of rule 14 is

$$
x_{14}^{1}=\sum_{i=0}^{I} \delta\left[-2 x_{i-1}+x_{i}+x_{i+1}-\frac{1}{2}\right] 2^{-(i+1)}
$$

where

$$
\delta\{w\}= \begin{cases}1, & w>0 \\ 0, & w \leq 0\end{cases}
$$

In order to transform every concrete bit string into abstract decimal digit, we use characteristic function $x_{N}$. Next, we plot the characteristic function diagram for rule 14 . We choose $I=14$. Figure 3 describes that all points fall into Bernoulli-shift map after eight iterations under rule 14, which shows that $\Lambda_{14}$ is global attractor of $f_{14}$. The phenomenon also shows that the prediction in Remark 3.22 is correct. Two sets $\Lambda_{1}^{14}$ and $\Lambda_{2}^{14}$ can also be found in Figure 3. The dots in the two straight lines, whose slope is $-1 / 2$, are in the set $\Lambda_{1}^{14}$; the dots in the two straight lines, whose slope is 2 , are in the set $\Lambda_{2}^{14}$.

Two isles of Eden are shown in Figure 4. We find that the bit string $y=(\underbrace{\ldots, 0,1, \ldots}_{\text {repetition of }(0,1)})$ is isle of Eden, when the length $|y|$ is even. Some attractors are shown in Figure 5. Figure 5(a) shows that the period of attractor is 1 ; Figure $5(b)$ shows that the period of attractor is 

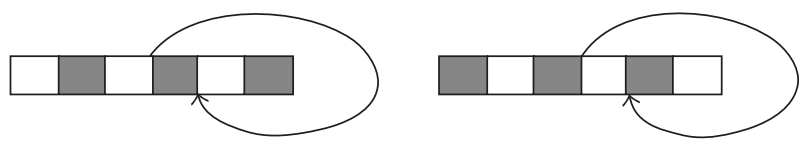

Figure 4: Two isles of Eden are shown, where $|x|=6$.

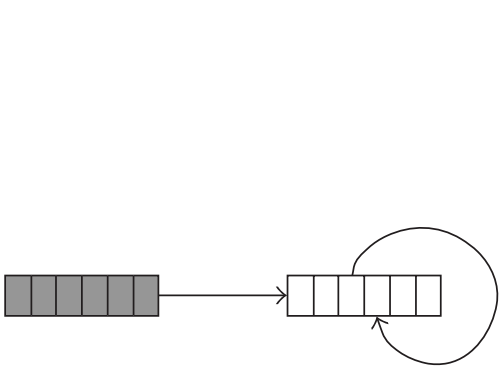

(a)

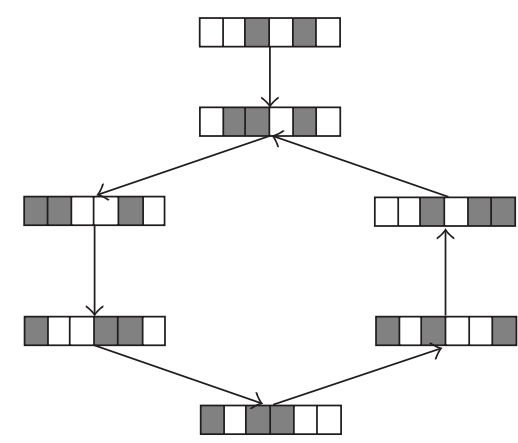

(c)

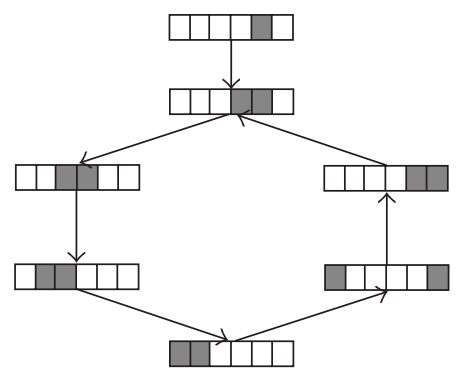

(b)

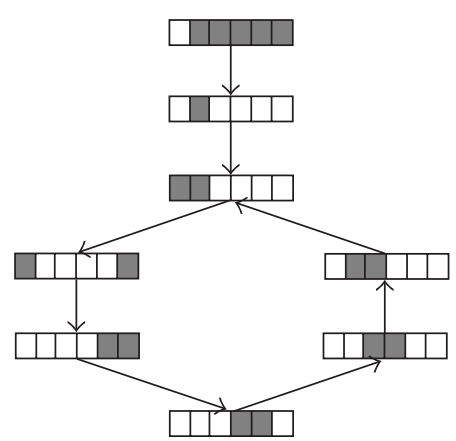

(d)

Figure 5: Some attractors of rule 14, where $I=5$, and the white pixel stands for 0 and black for 1 .

six, where $\beta=2, \sigma=1$, and $\tau=1$; Figure 5(c) shows that the period of attractor is six, where $\beta=-1 / 2, \sigma=-1$, and $\tau=1$; Figure 5(d) shows that after two iterations, the initial string is attracted. We find that the possible periods of attractors are 1 and 6 if $I=5$. By Proposition 3.5, the attractors of Figures 4 and 5(c) belong to $\Lambda_{1}^{14}$. By Proposition 3.17, the attractors of (a), (b), and (d) belong to $\Lambda_{2}^{14}$. There is a interesting phenomenon, that is, Figure 5(d), where one iteration accords with Proposition 3.5; however, the two iterations accord with Proposition 3.17, and the attractor belongs to $\Lambda_{2}^{14}$. The phenomenon shows that these two propositions can switch in some situation. The bit string of attractor in Figure 5(c) corresponds with strongly connected subgraph $r_{0} \rightarrow r_{2} \rightarrow r_{5} \rightarrow r_{4} \rightarrow r_{1} \rightarrow r_{3} \rightarrow r_{0}$ in Figure 1 .

Next, we use Lameray diagram [21] to present our attractors. The diagrams show clearly the iterative process of attractors. The evolution of characteristic function of period5 attractor is shown in Figure 6, where $I=4$, and the values of characteristic function are $0.0938,0.1875,0.3750,0.7500$, and 0.5313 , respectively. The five points fall into the time- 1 map $\left(\phi_{n}=\chi_{N}\left(\phi_{n-1}\right)\right)$ in Figure 5. Then we can associate this particular period-5 attractor of rule 14 as a period-5 point of a continuous map $f:[0,1] \rightarrow[0,1]$ which we know that it is chaotic because "period-5" implies chaos based on Li-York theorem [22] and Sarkovskii theorem [23]. According to Sarkovskii theorem, period-5 implies that the number of periods of $f_{14}$ are infinite. 


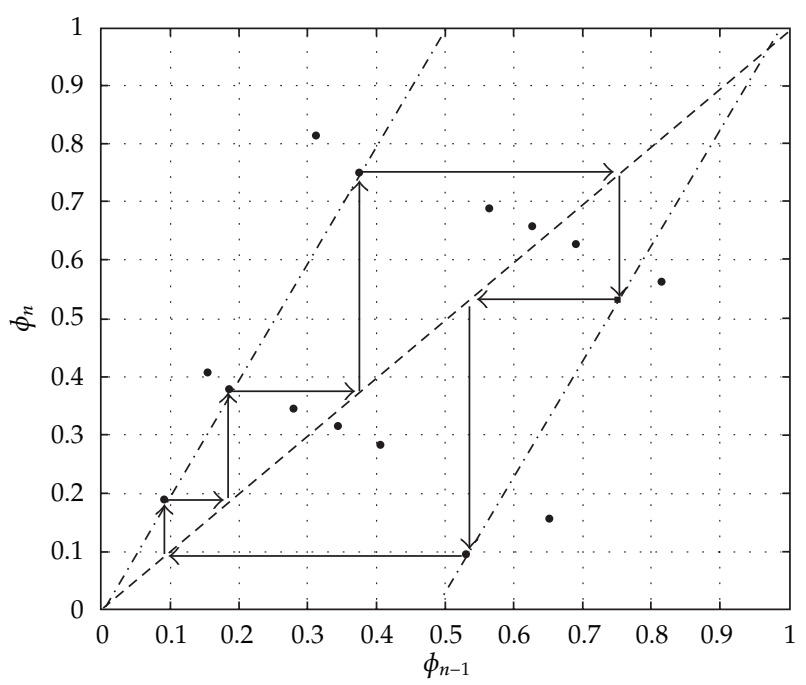

Figure 6: The evolution of characteristic function of the period- 5 attractor, where the values of characteristic function of the attractor are $0.0938,0.1875,0.3750,0.7500$, and 0.5313 , respectively.

In recent years, associative memory was researched in many papers [24, 25]. It is obvious that rule 14 can be used in associative memory. By strongly connected graph of rule 14 , we can get the elements on its attractors. Then, we can choose a bit string which belongs to an attractor as memory pattern. For example, bit string 110000 in Figure 5(b) can be chosen as a memory pattern. Since there are infinite orbits, the storage capability is very large. The associative memory model provides a solution to problem where time to recognize a pattern is independent of the number of patterns stored.

\section{Conclusion}

In this paper, the dynamical behaviors of rule 14 of global equivalence class $\varepsilon_{13}^{1}$ in ECA, where rule 14 is Bernoulli $\sigma_{\tau}$-shift rule, are carefully investigated from, the viewpoint of symbolic dynamics. We derive the conditions according to Bernoulli $\sigma_{\tau}$-shift evolution for rule 14. Then, we prove that rule 14 is chaotic in the sense of both Li-Yorke and Devaney on their attractors, respectively. We use diagrams to explain the attractor of rule 14, where characteristic function and Lameray diagram are used to describe that all points fall into Bernoulli-shift map after several iterations and to show clearly the iterative process of an attractor, respectively.

\section{Acknowledgments}

This work was supported in part by the National Natural Science Foundation of China under Grant 60973114, Grant 61170249, and Grant 61003247, in part by the Natural Science Foundation project of CQCSTC under Grant 2009BA2024, in part by the State Key Laboratory of Power Transmission Equipment \& System Security and New Technology, Chongqing University, under Grant 2007DA10512711206, and in part by Teaching \& Research Program of Chongqing Education Committee (KJ110401). 


\section{References}

[1] J. von Neumann, The General and Logical Theory of Automata, Pergamon Press, London, UK, 1951.

[2] H. Beigy and M. R. Meybodi, "Cellular learning automata with multiple learning automata in each cell and its applications," IEEE Transactions on Systems, Man, and Cybernetics B, vol. 40, no. 1, pp. 54-65, 2010.

[3] S. Nandi, B. K. Kar, and P. P. Chaudhuri, "Theory and applications of cellular automata in cryptography," IEEE Transactions on Computers, vol. 43, no. 12, pp. 1346-1357, 1994.

[4] F. Bao, "Cryptanalysis of a partially known cellular automata cryptosystem," IEEE Transactions on Computers, vol. 53, no. 11, pp. 1493-1497, 2004.

[5] Z. Eslami, S. H. Razzaghi, and J. Z. Ahmadabadi, "Secret image sharing based on cellular automata and steganography," Pattern Recognition, vol. 43, no. 1, pp. 397-404, 2010.

[6] R. J. Chen and S. J. Horng, "Novel SCAN-CA-based image security system using SCAN and 2-D von Neumann cellular automata," Signal Processing, vol. 25, no. 6, pp. 413-426, 2010.

[7] M. Tomassini and M. Perrenoud, "Cryptography with cellular automata," Applied Soft Computing, vol. 1, no. 2, pp. 151-160, 2001.

[8] S. Wolfram, "Universality and complexity in cellular automata," Physica D, vol. 10, no. 1-2, pp. 1-35, 1984.

[9] S. Wolfram, Theory and Applications of Cellular Automata, World Scientific, Singapore, 1986.

[10] S. Wolfram, A New Kind of Science, Wolfram Media, Inc., Champaign, Ill, USA, 2002.

[11] F. Y. Chen, W. F. Jin, G. R. Chen, F. F. Chen, and L. Chen, "Chaos of elementary cellular automata rule 42 of Wolfram's class II," Chaos, vol. 19, no. 1, Article ID 013140, 6 pages, 2009.

[12] F. F. Chen and F. Y. Chen, "Complex dynamics of cellular automata rule 119," Physica A, vol. 388, no. 6, pp. 984-990, 2009.

[13] L. Chen, F. Y. Chen, F. F. Chen, and W. F. Jin, "Complex symbolic dynamics of bernoulli shift cellular automata rule," in Proceedings of the 9th International Conference for Young Computer Scientists (ICYCS '08), pp. 2868-2873, 2008.

[14] Q. Han, X. Y. Liao, C. D. Li, and L. P. Feng, "Complex dynamics behaviors in cellular automata rule 35," Journal of Cellular Automata, vol. 6, no. 6, pp. 487-504, 2011.

[15] L. O. Chua, V. I. Sbitnev, and S. Yoon, "A nonlinear dynamics perspective of Wolfram's new kind of science. III. Predicting the unpredictable," International Journal of Bifurcation and Chaos in Applied Sciences and Engineering, vol. 14, no. 11, pp. 3689-3820, 2004.

[16] L. O. Chua, V. I. Sbitnev, and S. Yoon, "A nonlinear dynamics perspective of Wolfram's new kind of science. IV. From Bernoulli shift to $1 / f$ spectrum," International Journal of Bifurcation and Chaos in Applied Sciences and Engineering, vol. 15, no. 4, pp. 1045-1183, 2005.

[17] J. B. Guan, S. W. Shen, C. Tang, and F. Y. Chen, "Extending Chua's global equivalence theorem on Wolfram's new kind of science," International Journal of Bifurcation and Chaos in Applied Sciences and Engineering, vol. 17, no. 12, pp. 4245-4259, 2007.

[18] B. Kitchens, Symbolic Dynamics: One-Sided, Two-Sided and Countable State Markov Shifts, Springer, Berlin, Germany, 1990.

[19] Z. Zhou, Symbolic Dynamics, Shanghai Scientific and Technological Education Publishing House, Shanghai, China, 1997, (in Chinese).

[20] S. Amoroso and G. Cooper, "The garden-of-Eden theorem for finite configurations," Proceedings of the American Mathematical Society, vol. 26, pp. 158-164, 1970.

[21] L. P. Shilnikov, A. L. Shilnikow, D. V. Turaev, and L. O. Chua, Methods of Qualitative Theory in Nonlinear Dynamics: Part 1, World Scientific, Singapore, 1998.

[22] T. Y. Li and J. Yorke, "Period three implies chaos," The American Mathematical Monthly, vol. 82, no. 10, pp. 985-992, 1975.

[23] A. N. Sarkovskii, "Coexistence of the cycles of a continuous mapping of the line into itself," International Journal of Bifurcation and Chaos, vol. 5, pp. 1263-1273, 1995.

[24] P. Maji, N. Ganguly, and P. P. Chaudhuri, "Error correcting capability of cellular automata based associative memory," IEEE Transactions on Systems, Man, and Cybernetics A, vol. 33, no. 4, pp. 466-480, 2003.

[25] N. Ganguly, P. Maji, B. K. Sikdar, and P. P. Chaudhuri, "Design and characterization of cellular automata based associative memory for pattern recognition," IEEE Transactions on Systems, Man, and Cybernetics B, vol. 34, no. 1, pp. 672-679, 2004. 


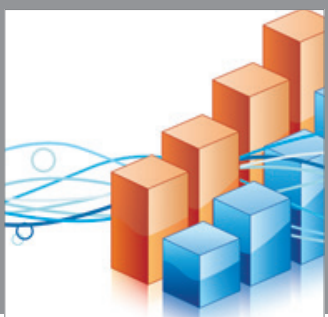

Advances in

Operations Research

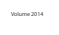

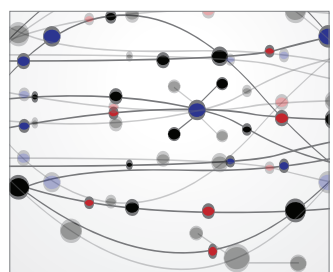

\section{The Scientific} World Journal
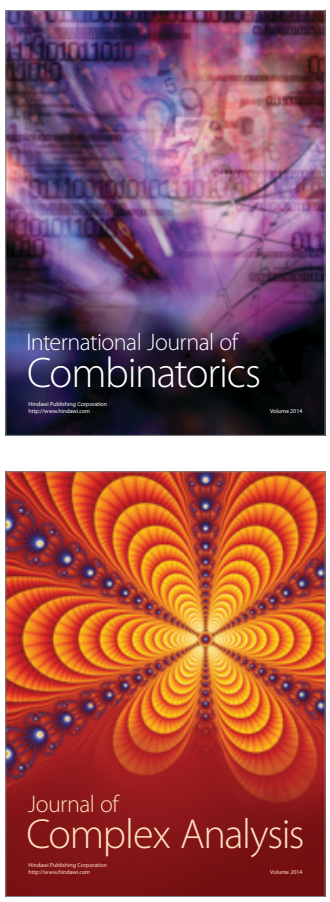

International Journal of

Mathematics and

Mathematical

Sciences
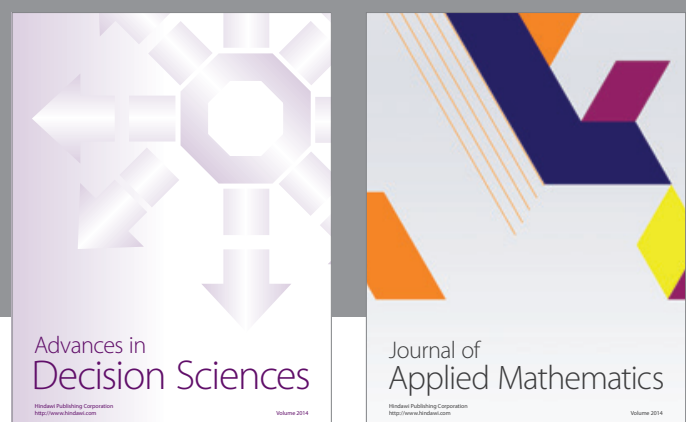

Journal of

Applied Mathematics
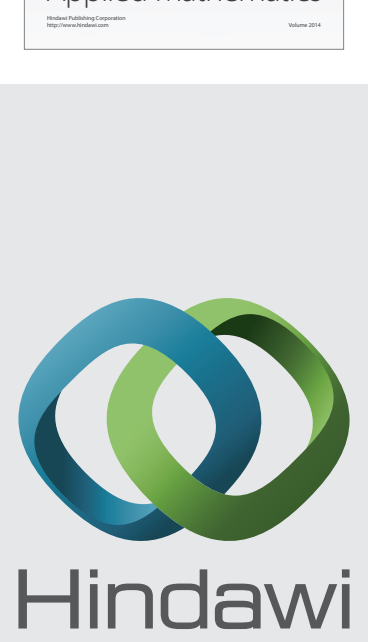

Submit your manuscripts at http://www.hindawi.com
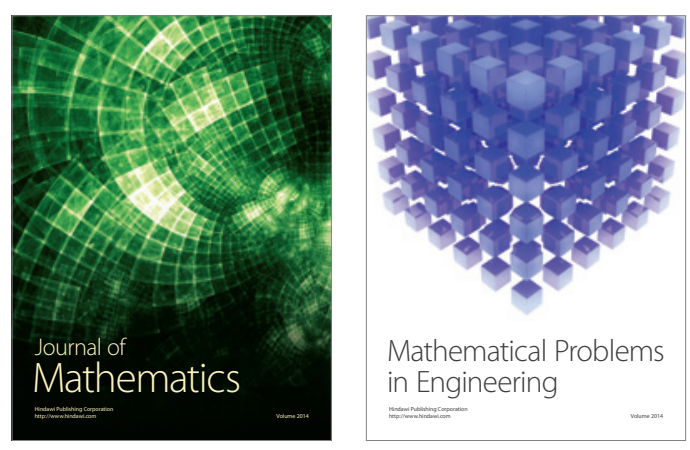

Mathematical Problems in Engineering
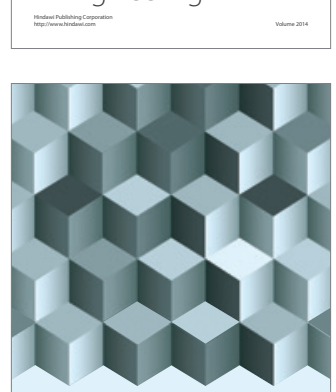

Journal of

Function Spaces
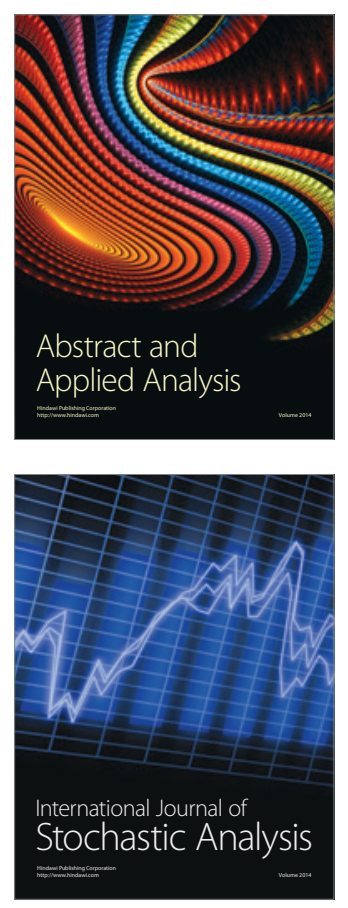

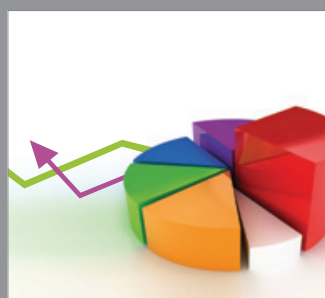

ournal of

Probability and Statistics

Promensencen
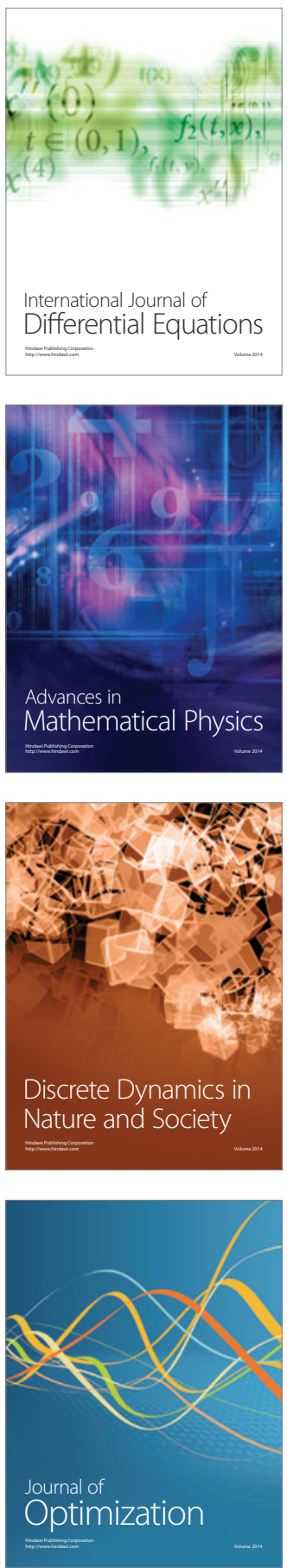